\title{
Semiclassical Density Functional Theory: Strutinsky Energy Corrections in Quantum Dots
}

\author{
Denis Ullmo, ${ }^{1}$ Tatsuro Nagano, ${ }^{2}$ Steven Tomsovic, ${ }^{2}$ and Harold U. Baranger ${ }^{3}$ \\ ${ }^{1}$ Laboratoire de Physique Théorique et Modèles Statistiques (LPTMS), 91405 Orsay Cedex, France \\ ${ }^{2}$ Department of Physics, Washington State University, Pullman, WA 99164-2814, USA \\ ${ }^{3}$ Department of Physics, Duke University, Box 90305, Durham, NC 27708-0305, USA
}

(18 July 2000)

\begin{abstract}
We develop a semiclassical density functional theory in the context of quantum dots. Coulomb blockade conductance oscillations have been measured in several experiments using nanostructured quantum dots. The statistical properties of these oscillations remain puzzling, however, particularly the statistics of spacings between conductance peaks. To explore the role that residual interactions may play in the spacing statistics, we consider many-body systems which include electron-electron interactions through an explicit density functional. First, we develop an approximate series expansion for obtaining the ground state using the idea of the Strutinsky shell correction method. Next, we relate the second-order semiclassical corrections to the screened Coulomb potential. Finally, we investigate the validity of the approximation method by numerical calculation of a one-dimensional model system, and show the relative magnitudes of the successive terms as a function of particle number.
\end{abstract}

PACS numbers: 73.23.Hk, 05.45.Mt, 71.10.Ca, 71.15.Mb

\section{INTRODUCTION}

A recurring problem in modern physics is how to add quantization effects to a basically successful macroscopic theory. This question arises particularly in the semiclassical regime - large quantum number-where the quantum effects are often corrections to the essentially classical macroscopic physics. Perhaps the best known example starts with the Thomas-Fermi theory of the atom [1], which is macroscopic in essence, and then evaluates the contribution of electronic shell structure to the ground state energy [1] 34. The very natural result that the shell contribution is given by the quantized levels of the self-consistent Thomas-Fermi potential has been used extensively 四 6 . However, it has only been in recent decades, starting with the work of V. M. Strutinsky [7 9], that a systematic way of answering the recurring general problem has been developed.

Our own immediate interest is in quantum dots - small electrically conducting regions in which the quantum properties of the confined electrons are important [10] - and our aim here is to treat quantum corrections to the ground state energy of these dots by further developing the Strutinsky method.

Quantum dots can be formed, for instance, by gate depletion of a two-dimensional electron gas (2DEG) in a GaAs-AlGaAs heterostructure. Because of the high quality of this material and interface, the mean free path of the electrons far exceeds the size of the quantum dot. One can view an electron as propagating ballistically within a confining potential created by electrostatic gates patterned on the surface of the heterostructure. For transport measurements, dots can be coupled weakly to leads; when the conductance of each lead falls below $2 e^{2} / h$, electron transport through the dot occurs only by tunneling, and the number of particles within the dot becomes quantized. In this regime, the conductance is suppressed due to the electrostatic energy associated with a localized charge [10], an effect known as the Coulomb blockade (first reported in 1951 by Gorter [1]]). When the dot potential is tuned by a gate voltage so that adding one electron costs no energy, a large conductance peak appears [10] - though the electrons must still tunnel from the leads, there is no additional electrostatic barrier to conduction. Sweeping such a gate voltage produces periodic Coulomb blockade oscillations of the conductance through dots $110,12,13]$.

The Coulomb blockade is a classical effect observable in a broad temperature range, $k_{B} T<e^{2} / C$, where $C$ is the total dot capacitance. Over most of this range, both the spacing and height of the peaks is constant- the spacing is $e^{2} / C$ and the height is given by the resistance of the two tunneling barriers acting in series. However, there is a low temperature regime below a few hundred millikelvin for which $k_{B} T<\Delta$, where $\Delta$ is the mean single-particle level spacing of the isolated dot. There, quantum interference and coherence become important [10]. The Coulomb blockade peaks grow as the temperature decreases, and novel fluctuation properties emerge 
involving both the peak heights [14 [16] and spacings [17 21]. The spacings give information about the ground state energies while the heights involve the magnitude of the wavefunction near the levels.

With some success, random-matrix-theory (RMT) based approaches 22 have been used in order first to predict [23] and then to explain the statistical properties found. In the simplest approximation [17], known as the constant interaction model, the ground state energy of the dot is expressed as

$$
E(N)=\frac{e^{2} N^{2}}{2 C}+\sum_{i=1}^{N} \epsilon_{i}
$$

where $N$ is the number of electrons in the dot and $\epsilon_{i}$ are single-particle energies. The first term is the classical charging energy; the second is the total energy of a system of non-interacting quasi-particles. Supposing that the single-particle classical dynamics within the dot is chaotic, the Bohigas-Giannoni-Schmit conjecture applies [24] and implies that the single-particle quantum properties follow RMT. It is well-established that RMT predicts repulsion amongst the $\epsilon_{i}$ and Gaussian random behavior in the eigenfunctions [25]. Within this model, the conductance peak height statistics are in good agreement with experimental results 14,15 after also incorporating non-zero temperature effects and interference modulations due to periodic paths coupled to the leads [23,26,27]. On the other hand, it is found [17 21] that the fluctuations in the peak spacings are considerably larger than the predictions [17,28,29], and there is no evidence for the level repulsion or electron spin degeneracy expected from a single-particle-like approach [30]. These discrepancies between the predictions of the constant interaction model and the observations point to the need for a quantum treatment of the electron-electron interactions, and, in particular, have triggered a number of studies based on Hartree-Fock calculations [31 33], or density functional theory in the local density approximation (LDA) [34, 35].

In nuclear physics, it has been recognized for some time that the dependence of nuclear ground state properties on the particle number can be viewed as the sum of two contributions, a "smooth part" which varies smoothly with the particle number and an "oscillatory behavior" producing the shell structure referred to as shell corrections. A similar decomposition is possible for any finite-size interacting fermion system. The smooth part comes basically from the bulk energy per unit volume integrated over the finite-size system, and the oscillating contributions come from quantum interference effects explicitly caused by the confinement. By supposing that the smooth part is known while the unknown oscillatory contribution is a correction, Strutinsky proposed in the 60's a physically motivated approach to, and an efficient way of calculating, the shell corrections [7].8].

Strutinsky's shell correction method is essentially a semiclassical approximation. It rests on the fact that the number of particles in the system considered is large, rather than on the interaction between the particles being weak. (One must, of course, work in a regime where the smooth starting point is basically valid.) Since the quantum dots in which we are interested contain on the order of a hundred electrons, they are a perfect place to apply the Strutinsky method. However, before doing so for a particular, realistic, two-dimensional geometry, we shall in this paper limit ourselves to a formal discussion of this method in conjunction with a one-dimensional illustrative example. In spite of the literature existing on this subject [9], we find it useful for two main purposes. First, the discussion and resulting expressions are noticeably simpler for quantum dots than for nuclei. This occurs because the existence of a smooth confining potential in the dot means that gradient corrections to the smooth density are not needed to confine the system at the zeroth-order (classical-like) approximation. The effect of these gradient terms can therefore be included in the first-order "shell" corrections, simplifying both the zeroth-order calculations (no gradient terms) and the first-order terms (no corrections to the Weyl part).

Our main purpose, however, is to take advantage of the fact that we use density functional theory rather than Hartree-Fock as a starting point, the former being presumably better suited to deal with the long-range Coulomb interaction present in quantum dots than the latter. This allows us to discuss in detail the second-order "residual interaction" terms of the Strutinsky method. By residual interaction we mean the weak interaction between Landau quasi-particles that comes from dressing the bare electron added to the quantum dot. In particular we will show, and illustrate, how these terms are related to the screened Coulomb interaction.

The remainder of the paper is organized as follows. The Thomas-Fermi and density functional theories are summarized in the next section, establishing our notation. Section IIII contains the Strutinsky method applied to density functional theory. This is the core of the paper; in particular, the relation of the second order terms to the screened Coulomb potential is derived. Section [V] recalls how the residual interaction terms contribute to conductance peak spacing distribution. Section $\mathrm{V}$ compares the whole approximation scheme to numerical calculations of a simplified model: interacting electrons in a one-dimensional quartic oscillator. Finally, we comment on the relationship between the Strutinsky development and the constant interaction model, and possible applications of the method. 


\section{DENSITY FUNCTIONAL THEORY}

The Hohenberg-Kohn theorem [36] states that for a system of interacting electrons in an external potential, $V_{\text {ext }}(\mathbf{r})$, there exists a functional, $\mathcal{F}_{\text {HKS }}[n]$, of the density of electrons, $n(\mathbf{r})$, such that: i) the density, $n_{g}(\mathbf{r})$, corresponding to the ground state of $N$ particles is an extremum of $\mathcal{F}_{\text {HKS }}[n]$ under the constraint that the total number of particles,

$$
N\left[n_{g}\right] \equiv \int d \mathbf{r} n_{g}(\mathbf{r})
$$

is fixed, and ii) $\mathcal{F}_{\mathrm{HKS}}\left[n_{g}\right]$ is the total energy of the system. The explicit form of the Hohenberg-Kohn-Sham functional is not known [36,37]. In practice, one must be satisfied with approximations. We describe here first a generalized Thomas-Fermi approach and, second, the case when an explicit form of the density functional is assumed.

\section{A. Generalized Thomas-Fermi Approximation ([Semi]Classical Level)}

It is convenient to view the density functional as the sum of three parts: a classical charge contribution, the kinetic energy, and the unknown exchange-correlation functional which accounts for the balance [37. The first part is simple: the energy of a system of classical charges confined by an external potential, $V_{\text {ext }}$, is

$$
\mathcal{E}[n]=\mathcal{E}_{\text {ext }}[n]+\mathcal{E}_{\text {coul }}[n]
$$

where

$$
\begin{aligned}
\mathcal{E}_{\text {ext }}[n] & =\int n(\mathbf{r}) V_{\text {ext }}(\mathbf{r}) d \mathbf{r} \\
\mathcal{E}_{\text {coul }}[n] & =\frac{e^{2}}{2} \iint \frac{n(\mathbf{r}) n\left(\mathbf{r}^{\prime}\right)}{\left|\mathbf{r}-\mathbf{r}^{\prime}\right|} d \mathbf{r} d \mathbf{r}^{\prime} .
\end{aligned}
$$

For the kinetic energy, in the Thomas-Fermi approach the Pauli exclusion principle is introduced semiclassically by employing the idea that one quantum state occupies a volume $(2 \pi \hbar)^{d}$ in phase space. This implies that if many electrons want to be at the same place, they can do so only by increasing their kinetic energy. This gives the Thomas-Fermi approximation to the kinetic energy part of the density functional, $\mathcal{T}_{\mathrm{TF}}[n]$, expressed as

$$
\begin{aligned}
& \nu(\epsilon)=\frac{1}{(2 \pi \hbar)^{d}} \int \Theta\left(\epsilon-\frac{\mathbf{p}^{2}}{2 m}\right) d \mathbf{p} \\
& t_{\mathrm{TF}}(n)=\int_{0}^{n} \epsilon(\nu) d \nu \\
& \mathcal{T}_{\mathrm{TF}}[n]=\int t_{\mathrm{TF}}(n(\mathbf{r})) d \mathbf{r}
\end{aligned}
$$

where $d$ is the dimensionality of the system, $\Theta$ is the Heaviside step function, $t_{\mathrm{TF}}$ is the kinetic energy density, and $\nu(\epsilon)$ is the number of states per unit volume with energy less than $\epsilon$. A factor 2 in $\nu(\epsilon)$ is required if the electron spin degeneracy is taken into account.

Finally, the effect of exchange and correlation is included through a term $\mathcal{E}_{\mathrm{xc}}[n]$. In practice, an explicit form for this functional must be taken. For example, if the electron density is a sufficiently slowly varying function of position, one can approximate $\mathcal{E}_{\mathrm{xc}}[n]$ by taking the exact results for the uniform electron gas at the local density, the well-known local density approximation (LDA).

Within this approximation, then, the density functional is

$$
\mathcal{F}_{\mathrm{GTF}}[n]=\mathcal{T}_{\mathrm{TF}}[n]+\mathcal{E}_{\text {tot }}[n]
$$

where

$$
\mathcal{E}_{\text {tot }}[n] \equiv \mathcal{E}_{\text {ext }}[n]+\mathcal{E}_{\text {coul }}[n]+\mathcal{E}_{\text {xc }}[n]
$$


The ground state energy and its electron distribution are obtained by minimizing $\mathcal{F}_{\text {GTF }}$ under the constraint (11), yielding the self-consistency equation

$$
\frac{\delta \mathcal{T}_{\mathrm{TF}}}{\delta n}\left[n_{\mathrm{GTF}}\right](\mathbf{r})+V_{\mathrm{eff}}\left[n_{\mathrm{GTF}}\right](\mathbf{r})=\mu_{\mathrm{GTF}}
$$

with the effective potential

$$
V_{\text {eff }}[n](\mathbf{r}) \equiv \frac{\delta \mathcal{E}_{\text {tot }}}{\delta n}[n](\mathbf{r})
$$

Notice that to make use of Eq. (7), one must have an explicit form for $\mathcal{E}_{\mathrm{xc}}$ in order to take the functional derivative in Eq. (8).

We call this approach "generalized-Thomas-Fermi" (GTF) because it uses the Thomas-Fermi approximation for the kinetic energy but retains macroscopic aspects of exchange and correlation. In particular, the short-range effects of exchange-correlation in a uniform system can be included.

\section{B. Kohn-Sham Equations (Quantum Mechanical Level)}

In standard implementations of density functional theory (DFT), the kinetic energy is treated quantum mechanically rather than (semi)classically as in GTF. To accomplish this, one introduces $N$ orthogonal functions $\left\{\phi_{1}(\mathbf{r}), \ldots, \phi_{N}(\mathbf{r})\right\}$ such that the electron density is defined as

$$
n(\mathbf{r}) \equiv \sum_{i=1}^{N}\left|\phi_{i}(\mathbf{r})\right|^{2}
$$

and the kinetic energy is

$$
\begin{aligned}
\mathcal{T}_{\text {DFT }}[n] & =\sum_{i=1}^{N}\left\langle\phi_{i}|\hat{T}| \phi_{i}\right\rangle \\
& =\frac{\hbar^{2}}{2 m} \int \sum_{i=1}^{N}\left|\nabla \phi_{i}(\mathbf{r})\right|^{2} d \mathbf{r} .
\end{aligned}
$$

Thus, the density functional becomes

$$
\mathcal{F}_{\mathrm{DFT}}[n]=\mathcal{T}_{\mathrm{DFT}}[n]+\mathcal{E}_{\text {tot }}[n] .
$$

To find the ground state energy, one minimizes $\mathcal{F}_{\mathrm{DFT}}$ with respect to the functions $\phi_{i}(\mathbf{r})$ under the constraints

$$
\int\left|\phi_{i}(\mathbf{r})\right|^{2} d \mathbf{r}=1, \quad i=1, \ldots, N
$$

The result is a Schrödinger equation

$$
\left(-\frac{\hbar^{2}}{2 m} \nabla^{2}+V_{\mathrm{eff}}[n](\mathbf{r})\right) \phi_{i}(\mathbf{r})=\epsilon_{i} \phi_{i}(\mathbf{r}), \quad i=1, \ldots, N
$$

where $\epsilon_{1}, \ldots, \epsilon_{N}$ are Lagrange multipliers and the effective potential is again defined by Eq. (8); these are the Kohn-Sham equations [37]. The Eqs. (8), (9), and (13) are the set of self-consistent equations for finding the electron density, $n_{\mathrm{DFT}}(\mathbf{r})$, and then the ground state energy $E_{\mathrm{DFT}}=\mathcal{F}_{\mathrm{DFT}}\left[n_{\mathrm{DFT}}\right]$.

As in the discussion of the GTF above, in order to actually solve the Kohn-Sham equations, an explicit form for the exchange-correlation functional is required. The simplest case is when $\mathcal{E}_{\mathrm{xc}}$ is an integral over space of a function (not functional) of the local density; this is the well-known local-density approximation (LDA). But other more complicated explicit forms are possible, for example the generalized gradient corrections to LDA [38. 


\section{APPROXIMATE GROUND STATE ENERGY: THE STRUTINSKY ENERGY-CORRECTION METHOD}

\section{A. Expansion of the DFT Ground State Energy}

In this section we develop an approximation to $E_{\mathrm{DFT}}\left[n_{\mathrm{DFT}}\right]$ starting from the solution of the generalizedThomas-Fermi equation, $n_{\mathrm{GTF}}$. The main motivation is to develop a physical interpretation of the difference between these two approaches in finding the ground state energy. In addition, the approximation is of interest numerically for large problems since it involves a self-consistent solution of only the GTF equation rather than the more involved Kohn-Sham equations. We use the method introduced by V. M. Strutinsky [7,8] originally in the context of a Hartree-Fock rather than density-functional approach. His method describes the interacting system self consistently, first with the quantum interference effects turned off, and then by introducing them perturbatively. As discussed in the introduction, the idea is to add the "oscillatory" effects caused by interference in the confined system to a "smooth" essentially macroscopic description - these effects are essentially the Friedel oscillations [39] familiar in the context of impurities or surfaces.

To study the role of quantum interference effects in the DFT ground state energy, we will first show that the generalized-Thomas-Fermi result is a [semi]classical approximation to the DFT energy. The GTF approximation does, of course, contain some quantum mechanics - notably the Pauli exclusion principle which gives rise to the Fermi surface - and so is not truly classical. But only the simplest local quantum effects are present in GTF rather than the effects of interfering paths that one expects in a true semiclassical theory, hence our characterization of GTF as "[semi]classical".

To see this clearly, we introduce a convenient notation adapted from the semiclassical treatment of singleparticle problems: it is customary there to express the density of states as a sum of a smooth term slowly varying in energy, called the Weyl part, and a term which varies rapidly in energy (on the scale of the mean level separation), called the oscillatory part [40]. For a system governed by the Hamiltonian $H[V] \equiv \mathbf{p}^{2} / 2 m+V(\mathbf{r})$, where the potential is as yet unspecified, one can define the probability density of $N$ independent particles

$$
n[V](\mathbf{r})=\sum_{i=1}^{N}\left|\phi_{i}(\mathbf{r})\right|^{2}
$$

in terms of the eigenstates $\left\{\phi_{i}\right\}$ of $H$. We also define the Weyl part of $n[V]$ by

$$
n^{W}[V](\mathbf{r}) \equiv \frac{1}{(2 \pi \hbar)^{d}} \int \Theta\left[\mu^{W}-\mathbf{p}^{2} / 2 m-V(\mathbf{r})\right] d \mathbf{p}
$$

where $\mu^{W}$ must be chosen so that $N=\int n^{W}(\mathbf{r}) d \mathbf{r}$. Note that $n^{W}[V](\mathbf{r})$ is smooth in that it neglects quantum fluctuations in much the same way that the GTF approximation does. With this notation, one can derive the useful relation

$$
\frac{\delta \mathcal{T}_{\mathrm{TF}}}{\delta n}\left[n^{W}[V]\right](\mathbf{r})+V(\mathbf{r})=\mu^{W}
$$

Indeed, using

$$
\frac{\delta \mathcal{T}_{\mathrm{TF}}}{\delta n}[n](\mathbf{r})=\epsilon(n(\mathbf{r}))
$$

Eq. (16) reads $\epsilon\left(n^{W}[V](\mathbf{r})\right)=\mu^{W}-V(\mathbf{r})$. Applying the function $\nu$ introduced in Eq. (价) to both sides of the equality gives the definition of $n^{W}[V](\mathbf{r})$, Eq. (15).

Recalling that $V_{\text {eff }}[n]$ is defined as the variational derivative of $\mathcal{E}_{\text {tot }}$ (to be completely clear, it is not the inverse of $n\left[V_{\text {eff }}\right]$ ), we see that the self-consistency equation (9) which defines $n_{\mathrm{DFT}}$ is

$$
n_{\mathrm{DFT}}(\mathbf{r})=n\left[V_{\mathrm{eff}}\left[n_{\mathrm{DFT}}\right]\right](\mathbf{r}) .
$$

Similarly, Eq. (7) which defines $n_{\mathrm{GTF}}$ can, in applying the above prescription, be put in the form

$$
n_{\mathrm{GTF}}(\mathbf{r})=n^{W}\left[V_{\mathrm{eff}}\left[n_{\mathrm{GTF}}\right]\right](\mathbf{r}) .
$$


These equations do not signify that $n_{\mathrm{GTF}}$ is the Weyl part of $n_{\mathrm{DFT}}$; however, they do indicate that if one neglects the quantum interference terms (i.e. the difference between the exact particle density and its Weyl part), then the definitions of $n_{\mathrm{GTF}}$ and $n_{\mathrm{DFT}}$ become equivalent. It is in this sense that $n_{\mathrm{GTF}}$ is the [semi]classical approximation of $n_{\mathrm{DFT}}$.

Supposing $n_{\mathrm{GTF}}(\mathbf{r})$ and $E_{\mathrm{GTF}}=\mathcal{F}_{\mathrm{GTF}}\left[n_{\mathrm{GTF}}\right]$ known, we now seek to evaluate the corrections to the ThomasFermi energy,

$$
\Delta E \equiv E_{\mathrm{DFT}}-E_{\mathrm{GTF}}
$$

up to second order in

$$
\delta n \equiv n_{\mathrm{DFT}}-n_{\mathrm{GTF}} .
$$

For this purpose, we first introduce the quantities

$$
\begin{aligned}
\tilde{n}(\mathbf{r}) & \equiv n\left[V_{\mathrm{eff}}\left[n_{\mathrm{GTF}}\right]\right](\mathbf{r}) \\
\tilde{n}^{W}(\mathbf{r}) & \equiv n^{W}\left[V_{\mathrm{eff}}\left[n_{\mathrm{GTF}}\right]\right](\mathbf{r})=n_{\mathrm{GTF}}(\mathbf{r}) \\
\tilde{n}^{\mathrm{osc}}(\mathbf{r}) & =\tilde{n}(\mathbf{r})-\tilde{n}^{W}(\mathbf{r}) .
\end{aligned}
$$

Note that once $V_{\mathrm{eff}}\left[n_{\mathrm{GTF}}\right]$ is known, all of these can be computed through the diagonalization of the known single particle GTF Hamiltonian. As is well-known, the sum of the eigenvalues of the Kohn-Sham equations, $\mathcal{E}_{1 \mathrm{p}}[V] \equiv \sum_{1}^{N} \epsilon_{i}$, does not give the total energy of the $N$ particles because of double counting of the interaction energy, but rather 41

$$
E_{\mathrm{DFT}}=\mathcal{E}_{1 \mathrm{p}}\left[V_{\mathrm{eff}}\left[n_{\mathrm{DFT}}\right]\right]-\int d \mathbf{r} V_{\mathrm{eff}}\left[n_{\mathrm{DFT}}\right](\mathbf{r}) n_{\mathrm{DFT}}(\mathbf{r})+\mathcal{E}_{\mathrm{tot}}\left[n_{\mathrm{DFT}}\right]
$$

To proceed further, we use the relation proved in the appendix

$$
\mathcal{E}_{1 \mathrm{p}}[V+\delta V]-\mathcal{E}_{1 \mathrm{p}}[V] \simeq \frac{1}{2} \int \delta V(\mathbf{r})\left(n(\mathbf{r})+n^{\prime}(\mathbf{r})\right) d \mathbf{r}
$$

where $n(\mathbf{r}) \equiv n[V](\mathbf{r})$ and $n^{\prime}(\mathbf{r}) \equiv n[V+\delta V](\mathbf{r})$ and which is correct through second order in the changes. Upon inserting $V=V_{\mathrm{eff}}\left[n_{\mathrm{GTF}}\right]$ and $V+\delta V=V_{\mathrm{eff}}\left[n_{\mathrm{DFT}}\right]$, and thus $n=\tilde{n}$ and $n^{\prime}=n_{\mathrm{DFT}}$, the first term on the right-hand-side of Eq. (23) becomes

$$
\mathcal{E}_{1 \mathrm{p}}\left[V_{\mathrm{eff}}\left[n_{\mathrm{DFT}}\right]\right]=\mathcal{E}_{1 \mathrm{p}}\left[V_{\mathrm{eff}}\left[n_{\mathrm{GTF}}\right]\right]+\frac{1}{2} \int d \mathbf{r} \delta V_{\mathrm{eff}}(\mathbf{r})\left(n_{\mathrm{DFT}}(\mathbf{r})+\tilde{n}(\mathbf{r})\right)
$$

where $\delta V_{\mathrm{eff}} \equiv V_{\mathrm{eff}}\left[n_{\mathrm{DFT}}\right]-V_{\mathrm{eff}}\left[n_{\mathrm{GTF}}\right]$. Similarly, the second term in $E_{\mathrm{DFT}}\left[n_{\mathrm{DFT}}\right]$ is

$$
\begin{aligned}
\int V_{\mathrm{eff}}\left[n_{\mathrm{DFT}}\right](\mathbf{r}) n_{\mathrm{DFT}}(\mathbf{r}) d \mathbf{r} & =\int V_{\mathrm{eff}}\left[n_{\mathrm{GTF}}\right](\mathbf{r}) n_{\mathrm{GTF}}(\mathbf{r}) d \mathbf{r} \\
& +\int V_{\mathrm{eff}}\left[n_{\mathrm{GTF}}\right](\mathbf{r}) \delta n(\mathbf{r}) d \mathbf{r} \\
& +\int \delta V_{\mathrm{eff}}(\mathbf{r}) n_{\mathrm{DFT}}(\mathbf{r}) d \mathbf{r}
\end{aligned}
$$

Finally, the third term is

$$
\mathcal{E}_{\mathrm{tot}}\left[n_{\mathrm{DFT}}\right]=\mathcal{E}_{\mathrm{tot}}\left[n_{\mathrm{GTF}}\right]+\int\left(V_{\mathrm{eff}}\left[n_{\mathrm{GTF}}\right](\mathbf{r})+\delta V_{\mathrm{eff}}(\mathbf{r}) / 2\right) \delta n(\mathbf{r}) d \mathbf{r}
$$

with corrections which are third order in $\delta n$.

Combining all the terms together, we obtain

$$
\begin{aligned}
E_{\mathrm{DFT}} & \simeq \mathcal{E}_{1 \mathrm{p}}\left[V_{\mathrm{eff}}\left[n_{\mathrm{GTF}}\right]\right]-\int n_{\mathrm{GTF}}(\mathbf{r}) V_{\mathrm{eff}}\left[n_{\mathrm{GTF}}\right](\mathbf{r}) d \mathbf{r}+\mathcal{E}_{\mathrm{tot}}\left[n_{\mathrm{GTF}}\right] \\
& +\frac{1}{2} \int \delta V_{\mathrm{eff}}(\mathbf{r}) \tilde{n}^{\mathrm{osc}}(\mathbf{r}) d \mathbf{r} .
\end{aligned}
$$


In order to express directly the difference between the DFT and GTF ground states, it is convenient to use

$$
E_{\mathrm{GTF}}\left[n_{\mathrm{GTF}}\right]=\mathcal{T}_{\mathrm{TF}}\left[n_{\mathrm{GTF}}\right]+\mathcal{E}_{\mathrm{tot}}\left[n_{\mathrm{GTF}}\right]
$$

for $\mathcal{E}_{\text {tot }}$ In order to simplify the last term in Eq. (28), note that $\tilde{n}^{\text {osc }}$ is of order $\delta n$, and that therefore one only needs the first order variation of the effective potential, $\delta V_{\text {eff }}(\mathbf{r})=\int\left(\delta V_{\text {eff }} / \delta n\right)\left[n_{\mathrm{GTF}}\right]\left(\mathbf{r}, \mathbf{r}^{\prime}\right) \delta n\left(\mathbf{r}^{\prime}\right) d \mathbf{r}^{\prime}$, to obtain $E_{\mathrm{DFT}}$ correct through second order. Thus the final expression for the Strutinsky energy correction is

$$
E_{\mathrm{DFT}} \simeq E_{\mathrm{GTF}}+\Delta E^{(1)}+\Delta E^{(2)}
$$

where the first and second order correction terms are

$$
\begin{aligned}
\Delta E^{(1)} & =\mathcal{E}_{1 \mathrm{p}}\left[V_{\mathrm{eff}}\left[n_{\mathrm{GTF}}\right]\right]-\int n_{\mathrm{GTF}}(\mathbf{r}) V_{\mathrm{eff}}\left[n_{\mathrm{GTF}}\right](\mathbf{r}) d \mathbf{r}-\mathcal{T}_{\mathrm{TF}}\left[n_{\mathrm{GTF}}\right] \\
\Delta E^{(2)} & =\frac{1}{2} \iint \tilde{n}^{\mathrm{osc}}(\mathbf{r}) \frac{\delta V_{\mathrm{eff}}}{\delta n}\left[n_{\mathrm{GTF}}\right]\left(\mathbf{r}, \mathbf{r}^{\prime}\right) \delta n\left(\mathbf{r}^{\prime}\right) d \mathbf{r} d \mathbf{r}^{\prime} .
\end{aligned}
$$

In this approach, the DFT ground state energy is, then, the sum of a classical contribution-the generalizedThomas-Fermi result $E_{\mathrm{GTF}}$ - and two quantum contributions- $\Delta E^{(1)}$ and $\Delta E^{(2)}$. We now discuss and interpret these two correction terms.

\section{B. Interpretation of the First-Order Corrections}

The first-order correction is simply the oscillatory part of the single particle energy for a system of $N$ electrons evolving in the potential $V_{\mathrm{eff}}\left[n_{\mathrm{GTF}}\right]$. Indeed, the Weyl part of $\mathcal{E}_{1 \mathrm{p}}[V]$ is

$$
\mathcal{E}_{1 \mathrm{p}}^{W}[V] \equiv \frac{1}{(2 \pi \hbar)^{d}} \int\left(\frac{\mathbf{p}^{2}}{2 m}+V(\mathbf{r})\right) \Theta\left(\mu^{W}-\frac{\mathbf{p}^{2}}{2 m}-V(\mathbf{r})\right) d \mathbf{p} d \mathbf{r}
$$

where $\mu^{W}$ is fixed by $N=\int n^{W}[V](\mathbf{r}) d \mathbf{r}$. Separately integrating the kinetic and potential energy terms for $V=V_{\mathrm{eff}}\left[n_{\mathrm{GTF}}\right]$, one obtains

$$
\mathcal{E}_{1 \mathrm{p}}^{W}\left[V_{\mathrm{eff}}\left[n_{\mathrm{GTF}}\right]\right]=\int d \mathbf{r} \int_{0}^{\mu^{W}-V_{\mathrm{eff}}\left[n_{\mathrm{GTF}}\right](\mathbf{r})} \epsilon \frac{d \nu}{d \epsilon} d \epsilon+\int n^{W}\left[V_{\mathrm{eff}}\left[n_{\mathrm{GTF}}\right]\right](\mathbf{r}) V_{\mathrm{eff}}\left[n_{\mathrm{GTF}}\right](\mathbf{r}) d \mathbf{r} .
$$

In the first term one recognizes the Thomas-Fermi kinetic energy, $\mathcal{T}_{\mathrm{TF}}\left[n_{\mathrm{GTF}}\right]$, while in the second term $n^{W}\left[V_{\mathrm{eff}}\left[n_{\mathrm{GTF}}\right]\right]=n_{\mathrm{GTF}}$. Thus the first-order Strutinsky correction is

$$
\Delta E^{(1)}=\mathcal{E}_{1 \mathrm{p}}\left[V_{\mathrm{eff}}\left[n_{\mathrm{GTF}}\right]\right]-\mathcal{E}_{1 \mathrm{p}}^{W}\left[V_{\mathrm{eff}}\left[n_{\mathrm{GTF}}\right]\right] \equiv \mathcal{E}_{1 \mathrm{p}}^{\mathrm{osc}}\left[V_{\mathrm{eff}}\left[n_{\mathrm{GTF}}\right]\right] .
$$

The leading quantum corrections to GTF are found, then, by quantizing the single-particle levels in the GTF self-consistent potential: this is a very natural result which, in fact, was used extensively in atomic and nuclear physics [3 6] before it was first justified by Strutinsky [7,8].

\section{Interpretation of the Second-Order Corrections}

The second-order correction, Eq. (32), requires further work: this form is not useful because it expresses $\Delta E^{(2)}$ as a function of the unknown $\delta n$. A second equation is necessary for us to determine $\delta n$. Note that this is not the case for $\Delta E^{(1)}$ which is written completely in terms of $n_{\mathrm{GTF}}$.

The required second equation is obtained by relating $\delta n$ to the oscillatory part of $\tilde{n} \equiv n\left[V_{\mathrm{eff}}\left[n_{\mathrm{GTF}}\right]\right]$ which, of course, is known since it depends only on $n_{\mathrm{GTF}}$. We start with the two equations

$$
\begin{aligned}
& \frac{\delta \mathcal{T}_{\mathrm{TF}}}{\delta n}\left[n_{\mathrm{GTF}}\right]+V_{\mathrm{eff}}\left[n_{\mathrm{GTF}}\right]=\mu_{\mathrm{GTF}} \\
& \frac{\delta \mathcal{T}_{\mathrm{TF}}}{\delta n}\left[n_{\mathrm{DFT}}^{W}\right]+V_{\mathrm{eff}}\left[n_{\mathrm{DFT}}\right]=\mu_{\mathrm{DFT}}^{W} .
\end{aligned}
$$


The first equation here is the definition of $n_{\mathrm{GTF}}$, and the second one follows directly from the general relation (16). Now expand $n_{\mathrm{DFT}}$ about $n_{\mathrm{GTF}}$ in the second equation and subtract the first one from it. In the term involving $V_{\text {eff }}, \delta n$ appears. However, in the kinetic energy term, the density difference is $n_{\mathrm{DFT}}^{W}-n_{\mathrm{GTF}}=$ $\left(n_{\mathrm{DFT}}-n_{\mathrm{GTF}}\right)-\left(n_{\mathrm{DFT}}-n_{\mathrm{DFT}}^{W}\right)=\delta n-n_{\mathrm{DFT}}^{\mathrm{osc}}$. To close the equation we must relate $n_{\mathrm{DFT}}^{\mathrm{osc}}$ to $n_{\mathrm{GTF}}$. This is possible because in an equation for $\delta n$, which is by definition first order in corrections, only the first order part of the other quantities need be kept. Thus, we can approximate $n_{\mathrm{DFT}}^{\text {osc }}$ by similarly expanding $n_{\mathrm{DFT}}$ about $n_{\mathrm{GTF}}$, yielding

$$
n_{\mathrm{DFT}}^{\mathrm{osc}}=\left(n_{\mathrm{DFT}}-n_{\mathrm{DFT}}^{W}\right) \simeq\left(\tilde{n}-n_{\mathrm{GTF}}\right)=\tilde{n}^{\mathrm{osc}} .
$$

The combination of these results gives the closure equation

$$
\int d \mathbf{r} \frac{\delta V_{\mathrm{eff}}}{\delta n}\left(\mathbf{r}, \mathbf{r}^{\prime}\right) \delta n\left(\mathbf{r}^{\prime}\right)+\int d \mathbf{r} \frac{\delta^{2} \mathcal{T}_{\mathrm{TF}}}{\delta n^{2}}\left(\mathbf{r}, \mathbf{r}^{\prime}\right)\left(\delta n\left(\mathbf{r}^{\prime}\right)-\tilde{n}^{\mathrm{osc}}\left(\mathbf{r}^{\prime}\right)\right)=\Delta \mu
$$

where $\Delta \mu \equiv \mu_{\mathrm{DFT}}^{W}-\mu_{\mathrm{GTF}}$ is fixed by the condition $\int \delta n(\mathbf{r}) d \mathbf{r}=0$. This is an integral equation for $\delta n$ in terms of GTF quantities. If a numerical calculation of $\Delta E^{(2)}$ is needed, the computational cost is relatively modest, largely the inversion of an operator.

One obtains a very natural interpretation of the second-order correction (32) by using this closure equation. Consider the generalized-Thomas-Fermi problem, Eq. (36), and suppose the external potential is slightly modified by the quantity $\delta V_{\text {ext }}(\mathbf{r})$. One thus obtains a new solution of the GTF equation $n_{\mathrm{GTF}}^{\prime}=n_{\mathrm{GTF}}+\delta n_{\mathrm{GTF}}$ which would verify

$$
\frac{\delta \mathcal{T}_{\mathrm{TF}}}{\delta n}\left[n_{\mathrm{GTF}}^{\prime}\right]+V_{\mathrm{eff}}\left[n_{\mathrm{GTF}}^{\prime}\right]+\delta V_{\mathrm{ext}}=\mu_{G T F}^{\prime} .
$$

Subtracting Eq. (36) as before yields

$$
\int d \mathbf{r} \frac{\delta^{2} \mathcal{T}_{\mathrm{TF}}}{\delta n^{2}}\left(\mathbf{r}, \mathbf{r}^{\prime}\right) \delta n_{\mathrm{GTF}}\left(\mathbf{r}^{\prime}\right)+\int d \mathbf{r} \frac{\delta V_{\mathrm{eff}}}{\delta n}\left(\mathbf{r}, \mathbf{r}^{\prime}\right) \delta n_{\mathrm{GTF}}\left(\mathbf{r}^{\prime}\right)+\delta V_{\mathrm{ext}}=\Delta \mu .
$$

If we now choose the variation of the potential to be

$$
\delta V_{\mathrm{ext}}(\mathbf{r})=\int d \mathbf{r}^{\prime}\left(\delta^{2} \mathcal{E}_{\mathrm{tot}} / \delta n^{2}\right)\left[n_{\mathrm{GTF}}\right]\left(\mathbf{r}, \mathbf{r}^{\prime}\right) \tilde{n}^{\mathrm{osc}}\left(\mathbf{r}^{\prime}\right),
$$

$\delta n_{\mathrm{GTF}}+\tilde{n}^{\text {osc }}$ satisfies the same Eq. (39) as $\delta n$. This means that, at this level of approximation, $\delta n$ is the sum of $\tilde{n}^{\text {osc }}$ and the displacement of charges $\delta n_{\mathrm{GTF}}$ screening $\tilde{n}^{\text {osc }}$ in the GTF approximation. Indeed, the definition of a screened interaction $V_{\mathrm{sc}}$ implies

$$
\int d \mathbf{r}^{\prime} \frac{\delta V_{\text {eff }}}{\delta n}\left(\mathbf{r}, \mathbf{r}^{\prime}\right) \delta n\left(\mathbf{r}^{\prime}\right)=\int d \mathbf{r}^{\prime} V_{\mathrm{sc}}\left(\mathbf{r}, \mathbf{r}^{\prime}\right) \tilde{n}^{\text {osc }}\left(\mathbf{r}^{\prime}\right),
$$

and therefore that the second-order correction, Eq. [32, can be written

$$
\Delta E^{(2)}=\frac{1}{2} \int d \mathbf{r} d \mathbf{r}^{\prime} \tilde{n}^{\mathrm{osc}}(\mathbf{r}) V_{\mathrm{sc}}\left(\mathbf{r}, \mathbf{r}^{\prime}\right) \tilde{n}^{\mathrm{osc}}\left(\mathbf{r}^{\prime}\right) .
$$

Thus the second-order correction is simply the energy of interaction between the additional charge oscillations caused by the quantization, where the interaction is screened because, after all, the "other" electrons treated in GTF are around. Note that $V_{\mathrm{sc}}$ is the screened interaction within the finite sized system, not in the bulk, and so includes boundary effects [28]; under certain conditions, the bulk screened potential may be used [42]. More importantly, while the screened interaction here does include exchange-correlation at the GTF (macroscopic) level, the result (44) is a "direct-like" contribution while an "exchange-like" term is missed. This is related to the deficiencies of the LDA-like treatment of DFT here and presumably could be fixed through a local-spin-density functional approach. Such generalizations would be straight forward using the same arguments given here. 


\section{CONTRIBUTION OF THE RESIDUAL INTERACTION TO PEAK SPACING DISTRIBUTIONS}

As an example of the utility of the Strutinsky method for adding quantization effects to a macroscopic result, we turn to considering the spacing between peaks in the conductance through a quantum dot in the Coulomb blockade regime. The contribution of residual interactions have been estimated for chaotic systems within a random matrix theory framework 28, 43 45. There it was found to be small, but not too far from the scale necessary to explain the failure of the constant interaction model. Here our ultimate aim is to evaluate the effect of the residual interaction in specific model systems which often are not in a regime where their quantum properties have fully converged to the purely statistical behavior found in random matrix theory. Systems tend not to be purely chaotic, and even when chaotic, still exhibit manifestations of short time dynamics in their eigenproperties. This can often lead to important deviations from statistical limiting behaviors. We therefore briefly sketch the relationship between the residual interaction and the Coulomb blockade peak spacings.

The position of a conductance peak as a function of gate voltage is proportional to the change in the total energy of the system when an electron is added [12],

$$
\mu_{N}=E(N)-E(N-1)
$$

and the conductance peak spacing is proportional to the discrete inverse compressibility

$$
\begin{aligned}
\chi_{N} & =\mu_{N+1}-\mu_{N} \\
& =E(N+1)+E(N-1)-2 E(N) .
\end{aligned}
$$

For each of the ground state energies here we will insert the second-order Strutinsky approximation to the DFT energy. The first term, $E_{\mathrm{GTF}}$, is the ground state energy in the generalized-Thomas-Fermi approximation, and is essentially the charging energy of the dot. The first-order correction contains the single-particle quantization effects. In some sense these two terms together constitute the same level of approximation as the much used constant interaction model. In fact, more physics is included here since changes in the self-consistent confining potential [29] are explicitly contained in the Strutinsky approach [45], whereas due to the ad hoc nature of the constant interaction model, therein exists no information at all on the self-consistent potential. The second-order correction term, $\Delta E^{(2)}$, contains, then, the effects of the residual interaction.

\section{THE QUARTIC OSCILLATOR: A CASE STUDY}

Let us now illustrate the above approach with a particular example. For the sake of simplicity, we choose a one-dimensional model system consisting of $N$ electrons in the confining potential $V_{\text {ext }}(x)=x^{4} / 2$ with the interactions governed by the one-dimensional Poisson equation $d^{2} V_{\text {int }}[n](x) / d x^{2}=-4 \pi e^{2} n(x)$. This is a simple limit of a three-dimensional problem: the system is assumed to be invariant in the tranvserse directions $y$ and $z$ so that the interactions are between planes of charge, but the medium is extremely inhomogeneous with the transverse mass taken to infinity so that only one-dimensional quantum mechanics is needed. Exchange and correlation effects are turned off; thus the interaction functional is

$$
V_{\mathrm{int}}[n](x)=-2 \pi e^{2} \int_{-\infty}^{\infty} n\left(x^{\prime}\right)\left|x-x^{\prime}\right| d x^{\prime} .
$$

Note that use of the 1D Poisson equation causes an interaction which grows with distance. Use of the subscript "int" in this section, rather than "coul" above, is meant to distinguish this case from the three-dimensional Coulomb interaction. We emphasize that our interest in this simple model system is only as an illustration for better understanding of the Strutinsky method.

We vary the electron charge $e$ to see how well the Strutinsky scheme works for different strengths of the interaction, $e=0.5,1.0$ and 1.5 in units where $\hbar=m=1$. The electron spin degeneracy is not considered here. First, we perform generalized-Thomas-Fermi and density-functional-theory calculations directly. Next, using the GTF results, we apply the Strutinsky techniques to find approximate DFT results. Finally, these approximate results are compared to the actual DFT values. Because of the neglect of exchange-correlation here, the GTF approach reduces to true Thomas-Fermi and the DFT approach is simply the coupled Schrödinger-Poisson equations. 


\section{A. Thomas-Fermi Numerical Calculations}

For one-dimensional systems

$$
\nu(\epsilon)=\frac{1}{2 \pi \hbar} \int_{-\infty}^{\infty} \Theta\left(\epsilon-p^{2} / 2 m\right) d p=\frac{\sqrt{2 m \epsilon}}{\pi \hbar}
$$

and, thus, the kinetic energy term, Eq. (4), can be written explicitly as

$$
\mathcal{T}_{\mathrm{TF}}[n]=\frac{\pi^{2} \hbar^{2}}{6 m} \int_{-\infty}^{\infty} n(x)^{3} d x .
$$

The ground state density is obtained by solving the Thomas-Fermi equation [cf. Eq. (7)]

$$
\frac{\pi^{2} \hbar^{2}}{2 m} n_{\mathrm{GTF}}^{2}(x)+\frac{1}{2} x^{4}+V_{\mathrm{int}}\left[n_{\mathrm{GTF}}\right](x)=\mu_{\mathrm{GTF}}
$$

where we have used $\left(\delta T_{\mathrm{TF}} / \delta n\right)[n]=\epsilon(n)=\left(\pi^{2} \hbar^{2} / 2 m\right) n(x)^{2}$. By differentiating twice and using the Poisson equation, one obtains the second order differential equation

$$
\frac{\pi^{2} \hbar^{2}}{2 m} \frac{d^{2} n_{\mathrm{GTF}}^{2}(x)}{d x^{2}}+6 x^{2}-4 \pi e^{2} n_{\mathrm{GTF}}(x)=0 .
$$

This can then be transformed into coupled first-order equations

$$
\begin{aligned}
y_{1}(x) & =n_{\mathrm{GTF}}^{2}(x) \\
\frac{d y_{1}(x)}{d x} & =y_{2}(x) \\
\frac{d y_{2}(x)}{d x} & =\frac{2 m}{\pi^{2} \hbar^{2}}\left(4 \pi e^{2} \sqrt{y_{1}(x)}-6 x^{2}\right)
\end{aligned}
$$

which can be conveniently solved. Because of the symmetry of the system, $d n / d x=0$ at the origin and one need only specify the density at the origin as an initial condition. One repeats solving Eq. 52 ) adjusting $n_{\mathrm{GTF}}(x=0)$ on each iteration until the normalization condition $N=\int n_{\mathrm{GTF}}(x) d x$ is satisfied. Once the electron density $n_{\mathrm{GTF}}(x)$ is found, the ground state energy is obtained from

$$
E_{\mathrm{GTF}}=\mathcal{T}_{\mathrm{TF}}\left[n_{\mathrm{GTF}}\right]+\mathcal{E}_{\mathrm{ext}}\left[n_{\mathrm{GTF}}\right]+\mathcal{E}_{\mathrm{int}}\left[n_{\mathrm{GTF}}\right]
$$

where $\mathcal{T}_{\mathrm{TF}}$ is given in Eq (52) and

$$
\begin{aligned}
& \mathcal{E}_{\mathrm{ext}}\left[n_{\mathrm{GTF}}\right]=2 \int_{0}^{\infty} n_{\mathrm{GTF}}(x) \frac{1}{2} x^{4} d x \\
& \mathcal{E}_{\mathrm{int}}\left[n_{\mathrm{GTF}}\right]=2 \cdot \frac{1}{2} \int_{0}^{\infty} n_{\mathrm{GTF}}(x) V_{\mathrm{int}}\left[n_{\mathrm{GTF}}\right](x) d x .
\end{aligned}
$$

The electron densities $n_{\mathrm{GTF}}$ for $N=5,10$ and 20 with $e=1.0$ are plotted in Fig. 1(a) and the effective potential, $V_{\text {eff }}\left[n_{\mathrm{GTF}}\right]$ given by Eq. (8), in Fig. 1(b). All three cases show the same basic structure which can be simply understood as follows. Without the interaction $(e=0)$, the density would have one maximum at the origin since the external potential has a minimum at the center. Once the interaction is turned on, electrons repel each other and avoid the center, making two maxima in the density. Though not pictured, the larger the value of the electron charge $e$, the lower the central valley in the density, and the more the density maxima move away from the origin. As intuitively expected, the minimum points in the effective potential correspond to the maximum points of electron density, and increasing $e$ increases rapidly the bimodal nature of the density. 


\section{B. Quantum Numerical Calculations}

The numerical calculation of the DFT energy requires self-consistently solving

$$
\begin{aligned}
& \left(-\frac{\hbar^{2}}{2 m} \frac{d^{2}}{d x^{2}}+V_{\text {eff }}[n](x)\right) \phi_{i}(x)=\epsilon_{i} \phi_{i}(x) \\
& n(x)=\sum_{i=1}^{N}\left|\phi_{i}(x)\right|^{2} \\
& V_{\text {eff }}[n](x)=\frac{1}{2} x^{4}-2 \pi e^{2} \int_{-\infty}^{\infty} n\left(x^{\prime}\right)\left|x-x^{\prime}\right| d x^{\prime}
\end{aligned}
$$

which are the coupled Schrödinger-Poisson equations. We start the self-consistent iterations with the ThomasFermi potential $V_{\text {eff }}\left[n_{\mathrm{GTF}}\right]$. At each iteration, we first diagonalize the Hamiltonian $\hat{H}=\hat{\mathbf{p}}^{2} / 2 m+V_{\text {eff }}(\mathbf{r})$ expressed in the basis of $\hat{H}_{0}=\hat{\mathbf{p}}^{2} / 2+x^{4} / 2$. From the eigenvalues and eigenvectors of $\hat{H}\left[V_{\text {eff }}[n]\right]$, we can construct the electron density and the corresponding effective potential. Self-consistency is evaluated by comparing the effective potentials $V_{\text {eff }}^{\text {Old }}$ and $V_{\text {eff }}^{\text {New }}$ before and after each iteration (or equivalently the densities $n^{\text {Old }}$ and $n^{\text {New }}$ ).

Because of the well-known instability of the Poisson equation, one cannot simply use the output from one iteration, $V_{\mathrm{eff}}^{\mathrm{New}}$, as the input to the next 46. Instead, we feedback only part of the output

$$
V_{\text {eff }}^{\text {Old, Next Iteration }}=V_{\text {eff }}^{\text {Old }}+\alpha\left(V_{\text {eff }}^{\text {New }}-V_{\text {eff }}^{\text {Old }}\right), 0<\alpha<1
$$

where $\alpha$ is initially set as 0.5 . If the self-consistency is not improved, $\alpha$ is reduced by half and the iteration redone so that improvement is guaranteed for every iteration. We repeat this until the density reaches self-consistency,

$$
\max _{|x| \leq x_{\max }}\left|n^{\text {New }}(x)-n^{\text {OLD }}(x)\right| \leq 10^{-9} .
$$

We require self-consistency in the density rather than the potential because the overall magnitude of the density does not change significantly as $N$ increases.

Once the self-consistent density and effective potential are obtained, the Weyl part of the density, $n_{\text {DFT }}^{W}$, as well as the chemical potential $\mu_{\mathrm{DFT}}^{W}$ can be calculated from Eq. (15); the oscillating part of the density follows from $n_{\mathrm{DFT}}^{\mathrm{osc}}=n_{\mathrm{DFT}}-n_{\mathrm{DFT}}^{W}$. Finally, the self-consistent ground state energy is obtained using

$$
\mathcal{T}_{\mathrm{DFT}}\left[n_{\mathrm{DFT}}\right]=\mathcal{E}_{1 \mathrm{p}}\left[V_{\mathrm{eff}}\left[n_{\mathrm{DFT}}\right]\right]-2 \int_{0}^{\infty} n_{\mathrm{DFT}}(x) V_{\text {eff }}\left[n_{\mathrm{DFT}}\right](x) d x
$$

and the same expressions for $\mathcal{E}_{\text {ext }}$ and $\mathcal{E}_{\text {int }}$ as in the Thomas-Fermi calculation, Eq. (55). We have used the above relation for the kinetic energy instead of the definition since the eigenvalues are more stable than the eigenvectors in the numerical calculations.

The quantum electron densities for $N=5,10$, and 20 are superposed in Fig. 1(a) for electron charge $e=1.0$. One can see the quantum mechanical oscillations whose number of peaks corresponds to the electron number $N$. Note the decreasing oscillation amplitudes with increasing particle number, as well as the tunneling outside of the potential wall at the classical turning points. The effective potentials, superposed in Fig. 1(b), are indistinguishable from the corresponding Thomas-Fermi potentials.

\section{Strutinsky Energy Corrections}

In order to find the approximate ground state energy using the Strutinsky method, we start with the ThomasFermi density and potential, calculated above, and quantize in this potential by solving the Schrödinger equation $\left(-\frac{\hbar^{2}}{2 m} \frac{d^{2}}{d x^{2}}+V_{\mathrm{eff}}\left[n_{\mathrm{GTF}}\right]\right) \phi_{i}=\epsilon_{i} \phi_{i}$ for the eigenvalues and eigenvectors.

The first-order energy correction is given by Eq. (35); in our example, the expression for the Weyl part reduces to

$$
\mathcal{E}_{1 \mathrm{p}}^{W}\left[V_{\mathrm{eff}}\left[n_{\mathrm{GTF}}\right]\right]=2 \frac{\sqrt{2 m}}{3 \pi \hbar} \int_{0}^{\infty}\left(\mu^{W}+2 V_{\mathrm{eff}}\left[n_{\mathrm{GTF}}\right](x)\right) \sqrt{\mu^{W}-V_{\mathrm{eff}}\left[n_{\mathrm{GTF}}\right](x)} d x
$$


The second-order energy correction, from Eq. (32), is

$$
\begin{aligned}
\Delta E^{(2)} & =-\pi e^{2} \int_{-\infty}^{\infty} \int_{-\infty}^{\infty} \tilde{n}^{\mathrm{osc}}(x)\left|x-x^{\prime}\right| \delta n\left(x^{\prime}\right) d x d x^{\prime} \\
& =4 \pi e^{2} \int_{0}^{\infty} \tilde{n}^{\mathrm{osc}}(x)\left\{\int_{x}^{\infty}\left(x-x^{\prime}\right) \delta n\left(x^{\prime}\right) d x^{\prime}\right\} d x .
\end{aligned}
$$

The required input $\delta n$ follows from Eq. (39). This equation can be simplified by noting, first, for the kinetic energy term

$$
\frac{\delta^{2} \mathcal{T}_{\mathrm{TF}}}{\delta n^{2}}\left[n_{\mathrm{GTF}}\right]\left(x, x^{\prime}\right)=\frac{\delta \epsilon(\nu)}{\delta \nu}\left[n_{\mathrm{GTF}}\right] \delta\left(x-x^{\prime}\right) .
$$

Second, for the term depending on $V_{\text {eff }}$ note that

$$
\int_{-\infty}^{\infty} \frac{\delta V_{\mathrm{eff}}}{\delta n}\left[n_{\mathrm{GTF}}\right]\left(x, x^{\prime}\right) \delta n\left(x^{\prime}\right) d x^{\prime}=-2 \pi e^{2} \int_{-\infty}^{\infty} \delta n\left(x^{\prime}\right)\left|x-x^{\prime}\right| d x^{\prime}=V_{\mathrm{int}}[\delta n](x)
$$

implies

$$
\frac{d^{2} V_{\mathrm{int}}[\delta n](x)}{d x^{2}}=-4 \pi e^{2} \delta n(x) .
$$

Thus, by taking the second derivative with respect to $x$ of Eq. (39), one obtains

$$
\frac{\pi^{2} \hbar^{2}}{m} \frac{d^{2}}{d x^{2}}\left\{n_{\mathrm{GTF}}(x) \cdot\left(\delta n(x)-\tilde{n}^{\mathrm{osc}}(x)\right)\right\}-4 \pi e^{2} \delta n(x)=0 .
$$

This equation can be converted into the coupled first-order equations

$$
\begin{aligned}
y_{1}(x) & =n_{\mathrm{GTF}}(x) \cdot\left(\delta n(x)-\tilde{n}^{\mathrm{osc}}(x)\right) \\
\frac{d y_{1}(x)}{d x} & =y_{2}(x) \\
\frac{d y_{2}(x)}{d x} & =\frac{4 m e^{2}}{\pi \hbar^{2}}\left(\frac{y_{1}(x)}{n_{\mathrm{GTF}}(x)}+\tilde{n}^{\mathrm{osc}}(x)\right)
\end{aligned}
$$

which can be conveniently solved.

With the energy correction terms calculated, the Strutinsky scheme allows us to approximate the quantum ground state energy using essentially classical Thomas-Fermi quantities. We plot $\Delta E \equiv E_{\mathrm{DFT}}-E_{\mathrm{GTF}}, \Delta E-$ $\Delta E^{(1)}$, and $\Delta E-\Delta E^{(1)}-\Delta E^{(2)}$ as functions of $N$ to see the series convergence of the Strutinsky scheme in Figs. 2, 3 and 4 for $e=0.5,1.0$ and 1.5 respectively. In the first two cases, the convergence seems to be working well: for $e=0.5$, without correction terms the error is of order 0.1 and smooth, while the first-order correction term improves the accuracy to 0.001, and the second-order term to roughly 0.0001. For $e=1.0$, without correction terms the error is about 0.1, with the first-order correction the error reduces to 0.01, and with the second-order to 0.0004 .

For $e=1.5$, before comparing the order of magnitude of the different terms, it is useful to say a few words about the odd-even structure clearly visible in Fig. 4, and actually already apparent for $e=1.0$ at low $N$ in Fig. 3. The origin of this behavior is not related to spin (which has not been included) but can be readily understood by looking at the lower panel of Fig. 5 , which shows the effective Thomas-Fermi potential $V_{\mathrm{eff}}\left[n_{\mathrm{GTF}}\right]$ for $N=20, e=1.5$. Here, one sees that the latter has developed a barrier at the center of the well, the top of which lies very close to the chemical potential. For the quantum case, this means that the one particle levels below the Fermi energy are organized as quasi-doublets. This naturally leads to an odd-even effect since for $\mathrm{N}$ even (odd), the last occupied orbital has an energy consistently below (above) that suggested by the Weyl approximation.

Moreover, because the last occupied orbitals are close to the chemical potential and so near the top of the barrier, it is clear that semiclassical approximations will be "at risk" here. This is clearly seen for instance in Figs. 6 and 7, which, for coupling $e=1.5$ and $N=19$ and 20 particles, shows a comparison between the exact $\delta n(\mathbf{r}) \equiv n_{\mathrm{DFT}}(\mathbf{r})-n_{\mathrm{GTF}}(\mathbf{r})$ and its approximation obtained using Eq. (39). The two curves are almost on top 
of each other everywhere, except in the middle of the well - that is, near the maxima of the barrier. In addition, one observes that in that place, the approximation is worse for an odd than for an even number of particles. This can be explained by the fact that in the former case the last occupied orbital is symmetric and thus has a probability maximum at the origin, while in the latter case the last occupied orbital is antisymmetric and so goes to zero. As a consequence the errors in the Strutinsky approximation scheme also display an odd-even structure clearly seen in Fig. 5. If, however, one concentrates on the even case, where the effect of the central barrier is less important, without correction terms the error is about 0.2 , with the first-order correction the error is reduced to 0.02 , and further reduced to about 0.003 if one includes the second-order corrections.

\section{The Peak Spacings}

Turning now to the inverse compressibility $\chi_{N}$ introduced in Sect. IV, we observe the same trend as for the groundstate energy. The approximation $\chi_{N}^{\mathrm{STR}(0)}$, calculated strictly by the Thomas-Fermi approximation, already gives an excellent relative precision. Moreover, for $e=0.5$ and $e=1.0$, each term in the Strutinsky development significantly reduces the error (i.e. an order of magnitude or more for $N=18$ ). For these cases however the approximation is already much better than the mean spacing $\Delta$ if the first correction is included. We therefore show the data only for $e=1.5$, for which the corrections are enhanced by the proximity of the chemical potential to the top of the inner barrier. In Fig. 8(a), the $\chi_{N}$ are shown. The discrete points represent

the full quantum calculations and are taken as the reference points. It is seen that $\chi_{N}^{\operatorname{STR}(0)}$ does not capture the odd-even double-well effect, but otherwise captures the essential peak spacing behavior. In Fig. 8(b), the relative errors are shown as a function of $N$. More specifically, the difference between the quantum $\chi_{N}$ and one, two, or three terms of the Strutinsky series- $\chi_{N}^{\operatorname{STR}(0)}, \chi_{N}^{\operatorname{STR}(1)}$ and $\chi_{N}^{\operatorname{STR}(2)}$, respectively - is divided by $\Delta$, the mean level separation. It is seen that the majority of the odd-even, double-well effect is captured by the first correction term. Reassuringly, even in this case for which the inner barrier degrades the quality of the semiclassical development, each inclusion of an extra term in the series reduces somewhat the relative errors of the Strutinsky method. Moreover the improvement due to the addition of the second order correction becomes more significant with increasing $N$. Thus we see that even in this more difficult case, the Strutinsky method gives a excellent scheme of approximation in the semiclassical limit.

\section{CONCLUSION}

We have developed an approximate series expansion for studying the ground state of interacting systems using the idea of the Strutinsky shell correction method. We tested the validity of the Strutinsky scheme by numerical calculations of interacting electron systems in a one-dimensional, externally applied quartic potential. By varying the electron charge strength, we were able to confirm the stability of the method. It approximated extremely well the quantum mechanical DFT quantities using [semi]classical Thomas-Fermi data for three different charge strengths. One exceptional circumstance giving less reliable results was noted with respect to a barrier in $V_{\text {eff }}$ approaching the chemical potential. The calculations show a tendency for the series to converge as electron number increases. This is consistent with expectations for systems with large numbers of particles because of the increasing reliability of the Thomas-Fermi calculations as the system goes deeper into the semiclassical regime.

The method discussed in this paper could serve two purposes. On one hand, it gave us an efficient way to proceed with numerical calculations, and it is conceivable that this approach could be of some help for larger scale, realistic DFT calculations [34, 35, 38]. On the other hand, it also provides some physical insight by decomposing the total DFT energy into various contributions, each of them receiving an intuitive interpretation.

In the context of quantum dots, for instance, one of its simple but presumably useful applications could be to justify, and make more precise, the constant interaction model. Indeed, this latter is usually presented as an adhoc model motivated essentially by its simplicity. Here, up to some reinterpretation of what is the capacitance of the dot, we see that the constant interaction model can be understood as the first-order approximation in a Strutinsky development of a DFT calculation. One obtains, in addition, that the various parameters of the model (classical energy and potential governing the motion of the independent particles) are specified, and, in principle, can be computed explicitly. This makes it possible, for instance, to study the sensitivity of the dot's energy to the variation of an external parameter 45 . 
In the same way, the second-order correction term gives insight into the "residual" interactions between electrons. In the context of DFT it gives some basis to the fact that electrons in quantum dots behave as Landau quasi-particles interacting weakly through a screened Coulomb interaction. It moreover explicitly specifies how the screening process is affected by the confinement of the electrons, which might be relevant in the limit where the screening length is not much smaller than the size of the dots.

With the failure of the theoretical predictions of the single-particle constant-interaction model to explain the experimentally observed conductance peak spacing distributions [17 21], our original interest was to investigate what physical factors are missing and to understand better the statistical behaviors of quantum dots. We expect that the approach of defining the many-body system, explicitly including electron-electron interactions within density functional theory, to bring us closer to a resolution of the failure of the constant interaction model in this context. We leave application to more realistic systems for future work.

\section{ACKNOWLEDGMENTS}

We acknowledge helpful conversations with Oriol Bohigas, Matthias Brack, Nicholas Cerruti and Nicolas Pavloff. The LPTMS is an "Unité de recherche de l'Université Paris 11 associée au C.N.R.S." This work was supported in part by NSF grant \#PHY-9800106

\section{APPENDIX A:}

Consider a Hamiltonian $H=\mathbf{p}^{2} / 2 m+V(\mathbf{r})$ and its perturbed Hamiltonian $H^{\prime}=H+\delta V(\mathbf{r})$. We denote the eigenvalues and eigenvectors as $\epsilon_{i}$ and $\phi_{i}(\mathbf{r})\left[\epsilon_{i}^{\prime}\right.$ and $\left.\phi_{i}^{\prime}(\mathbf{r})\right]$ for $H\left(H^{\prime}\right)$.

To second order in $\delta V$, the perturbed eigenvalues are

$$
\epsilon_{i}^{\prime}=\epsilon_{i}+\epsilon_{i}^{(1)}+\epsilon_{i}^{(2)}
$$

where

$$
\begin{aligned}
\epsilon_{i}^{(1)} & =\left\langle\phi_{i}|\delta V| \phi_{i}\right\rangle \\
\epsilon_{i}^{(2)} & =\sum_{j \neq i} \frac{\left|\left\langle\phi_{i}|\delta V| \phi_{j}\right\rangle\right|^{2}}{\epsilon_{i}-\epsilon_{j}},
\end{aligned}
$$

assuming non-degenerate eigenstates.

Similarly, taking $H^{\prime}$ as the original Hamiltonian and $H=H^{\prime}-\delta V$ as the perturted Hamiltonian, one can write

$$
\epsilon_{i}=\epsilon_{i}^{\prime}+\epsilon_{i}^{\prime(1)}+\epsilon_{i}^{\prime(2)}
$$

where

$$
\begin{aligned}
& \epsilon_{i}^{\prime(1)}=-\left\langle\phi_{i}^{\prime}|\delta V| \phi_{i}^{\prime}\right\rangle \\
& \epsilon_{i}^{\prime(2)}=\epsilon_{i}^{(2)}+o\left(\delta V^{3}\right) .
\end{aligned}
$$

Subtracting Eq.(A3) from Eq.(A1), one obtains to second order in $\delta V$

$$
\begin{aligned}
\epsilon_{i}^{\prime}-\epsilon_{i} & =\frac{1}{2}\left(\epsilon_{i}^{(1)}-\epsilon_{i}^{\prime(1)}\right) \\
& =\frac{1}{2}\left(\left\langle\phi_{i}|\delta V| \phi_{i}\right\rangle+\left\langle\phi_{i}^{\prime}|\delta V| \phi_{i}^{\prime}\right\rangle\right) .
\end{aligned}
$$

Summing over $i$, one obtains

$$
\begin{aligned}
\mathcal{E}_{1 p}[V+\delta V]-\mathcal{E}_{1 p}[V] & =\frac{1}{2} \sum_{i=1}^{N}\left(\left\langle\phi_{i}|\delta V| \phi_{i}\right\rangle+\left\langle\phi_{i}^{\prime}|\delta V| \phi_{i}^{\prime}\right\rangle\right) \\
& =\frac{1}{2} \int \delta V(\mathbf{r})\left(n(\mathbf{r})+n^{\prime}(\mathbf{r})\right) d \mathbf{r} .
\end{aligned}
$$


[1] L. D. Landau and E. M. Lifshitz, Quantum Mechanis (Pergamon Press, New York, 1977) pp. 259-263, 275.

[2] N. H. March, Self-Consistent Field in Atoms: Hartree and Thomas-Fermi Atoms (Pergamon Press, New York, 1975).

[3] B.-G. Englert and J. Schwinger, Phys. Rev. A 32, 26 (1985).

[4] M. G. Mayer, Phys. Rev. 60, 184 (1941).

[5] R. Latter, Phys. Rev. 99, 510 (1955).

[6] O. Bohigas, X. Campi, H. Krivine, and J. Treiner, Phys. Lett. 64, 381 (1976).

[7] V. M. Strutinsky, Nucl. Phys. A122, 1 (1968).

[8] M. Brack, J. Damgaard, A. S. Jensen, H. C. Pauli, V. M. Strutinsky, and C. Y. Wong, Rev. Mod. Phys. 44, 320 (1972).

[9] For a recent review see M. Brack and R. K. Bhaduri, Semiclassical Physics (Addison-Wesley, Reading MA, 1997).

[10] For recent reviews see L. L. Sohn, L. P. Kouwenhoven, and G. Schön, editors, Mesoscopic Electron Transport (Kluwer Academic, Boston, 1997) and G. Timp, editor, Nanotechnology (Springer-Verlag, New York, 1999).

[11] C. J. Gorter, Physica 17, 777 (1951).

[12] C. W. J. Beenakker, Phys. Rev. B 44, 1646 (1991).

[13] H. van Houten, C. W. J. Beenakker, and A. A. M. Staring in Single Charge Tunneling Coulomb Blockade Phenomena in Nanostructures, edited by H. Grabert and M. H. Devoret (Plenum Press, New York, 1992) pp. 167-216.

[14] A. M. Chang, H. U. Baranger, L. N. Pfeiffer, K. W. West, and T. Y. Chang, Phys. Rev. Lett. 76, 1695 (1996).

[15] J. A. Folk, S. R. Patel, S. F. Godijn, A. G. Huibers, S. M. Cronenwett, and C. M. Marcus, Phys. Rev. Lett. 76, 1699 (1996).

[16] S. R. Patel, D. R. Stewart, C. M. Marcus, M. Gokcedag, Y. Alhassid, A. D. Stone, C. I. Duruos, and J. S. Harris, Jr., Phys. Rev. Lett. 815900 (1998).

[17] U. Sivan, R. Berkovits, Y. Aloni, O. Prus, A. Auerbach, and G. Ben-Yoseph, Phys. Rev. Lett. 77, 1123 (1996)

[18] S. R. Patel, D. R. Stewart, A. G. Huibers, C. M. Marcus, C. I. Duruos, J. S. Harris, Jr., K. Campman, and A. C. Gossard, Phys. Rev. Lett. 804522 (1998).

[19] F. Simmel, T. Heinzel, and D. A. Wharam, Europhys. Lett. 38, 123 (1997)

[20] F. Simmel, D. Abusch-Magder, D. A. Wharam, M. A. Kastner, and J. P. Kotthaus, Phys. Rev. B 59, R10441 (1999)

[21] S. Lüscher, T. Heinzel, K. Ensslin, W. Wegscheider, and M. Bichler, preprint arXiv:cond-mat/0002226.

[22] For a review of random matrix theory in the context of quantum transport see C. W. J. Beenakker, Rev. Mod. Phys. 69, 731 (1997).

[23] R. A. Jalabert, A. D. Stone, and Y. Alhassid, Phys. Rev. Lett. 68, 3468 (1992).

[24] O. Bohigas, M.-J. Giannoni, and C. Schmit, Phys. Rev. Lett. 52, 1 (1984); J. Physique Lett. 45, L-1015 (1984).

[25] T. A. Brody, J. Flores, J. B. French, P. A. Mello, A. Pandey, and S. S. M. Wong, Rev. Mod. Phys. 53, 385 (1981).

[26] Y. Alhassid, M. Gökcedag, and A. D. Stone, Phys. Rev. B 58, 7524 (1998).

[27] E. E. Narimanov, N. R. Cerruti, H. U. Baranger, and S. Tomsovic, Phys. Rev. Lett. 83, 2640 (1999); E. E. Narimanov, H. U. Baranger, N. R. Cerruti, and S. Tomsovic, submitted to Phys. Rev. B.

[28] Y. M. Blanter, A. D. Mirlin, and B. A. Muzykantskii, Phys. Rev. Lett. 78, 2449 (1997).

[29] R. O. Vallejos, C. H. Lewenkopf, and E. R. Mucciolo, Phys. Rev. Lett. 81, 677 (1998); Phys. Rev. B 60, 13682 (1999).

[30] D. R. Stewart, D. Sprinzak, C. M. Marcus, C. I. Duruoz, and J. S. Harris Jr., Science 278, 1784 (1997).

[31] P. N. Walker, G. Montambaux, Y. Gefen, Phys. Rev. Lett. 82, 5329 (1999); Phys. Rev. B 60, 2541 (1999).

[32] A. Cohen, K. Richter, and R. Berkovits Phys. Rev. B 60, 2536 (1999).

[33] K.-H. Ahn, K. Richter, and I.-H. Lee, Phys. Rev. Lett. 83, 4144 (1999).

[34] M. Stopa, Physica B 251, 228 (1998).

[35] K. Hirose, N. S. Wingreen Phys. Rev. B 59, 4604 (1999).

[36] P. Hohenberg and W. Kohn, Phys. Rev. 136, B 864 (1964).

[37] W. Kohn and L. J. Sham, Phys. Rev. 140, A 1133 (1965).

[38] P. Fulde, Electron Correlations in Molecules and Solids (Springer, New York, 1995) Chapter 3.

[39] J. Ziman, Principles of the Theory of Solids (Cambridge University Press, Cambridge, 1972) pp. 157-160.

[40] M. C. Gutzwiller, Chaos in Classical and Quantum Mechanics (Springer-Verlag, New York, 1990) pp. $254-275$.

[41] Note that even though Eq. (23) holds, $\mathcal{F}_{\text {DFT }}[n]$ is generally not equal to $F_{\mathrm{H}}[n] \equiv \mathcal{E}_{1 \mathrm{p}}\left[V_{\text {eff }}[n]\right]-\int d \mathbf{r} V_{\text {eff }}[n] n(\mathbf{r})+\mathcal{E}_{\text {tot }}[n]$ for an arbitrary density $n(\mathbf{r})$. Because of the fact that $\delta \mathcal{E}_{1 \mathrm{p}} / \delta V=n(r)$ [cf. Eq. 24]], it can nevertheless be shown that $\left(\delta F_{\mathrm{H}} / \delta n\right)\left[n_{\mathrm{DFT}}\right]=0 . F_{\mathrm{H}}$ is known as the Harris functional; see J. Harris, Phys. Rev. B 31, 1770 (1985).

[42] D. Ullmo, unpublished.

[43] P. W. Brouwer, Y. Oreg, and B. I. Halperin, Phys. Rev. B 60, R13977 (1999).

[44] H. U. Baranger, D. Ullmo, and L. I. Glazman, Phys. Rev. B 61, R2425 (2000).

[45] D. Ullmo and H. U. Baranger, in preparation.

[46] F. Herman and S. Skillman, Atomic Structure Calculations, (Prentice Hall, 1963). 


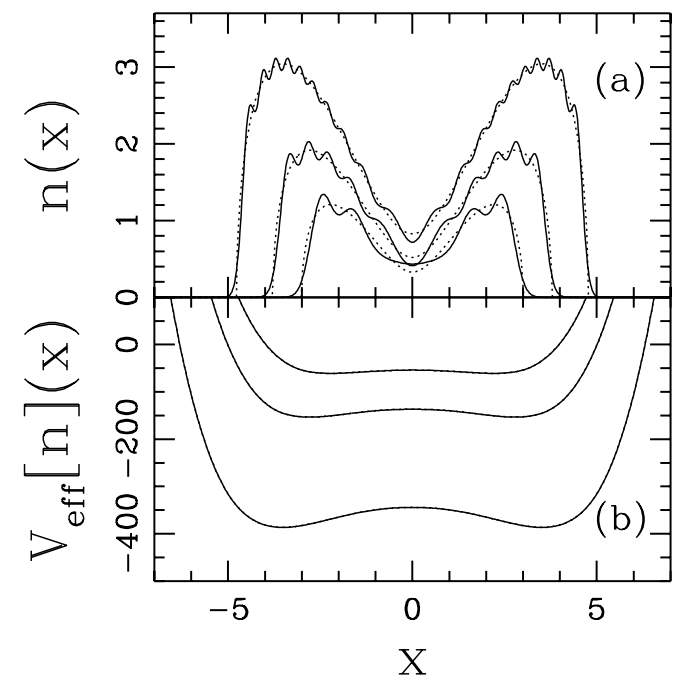

FIG. 1. The electron densities (upper panel) and effective potentials (lower panel) for interacting particles in a quartic potential. The results for both the Thomas-Fermi (dashed) and coupled Schrödinger-Poisson (solid) approximations are given. The electron charge is $e=1.0$, and the electron number is $N=5,10$, and 20 from bottom to top in upper panel, top to bottom in lower panel. $V_{\text {eff }}\left[n_{\mathrm{GTF}}\right]$ and $V_{\mathrm{eff}}\left[n_{\mathrm{DFT}}\right]$ coincide so well that one cannot distinguish the differences here.

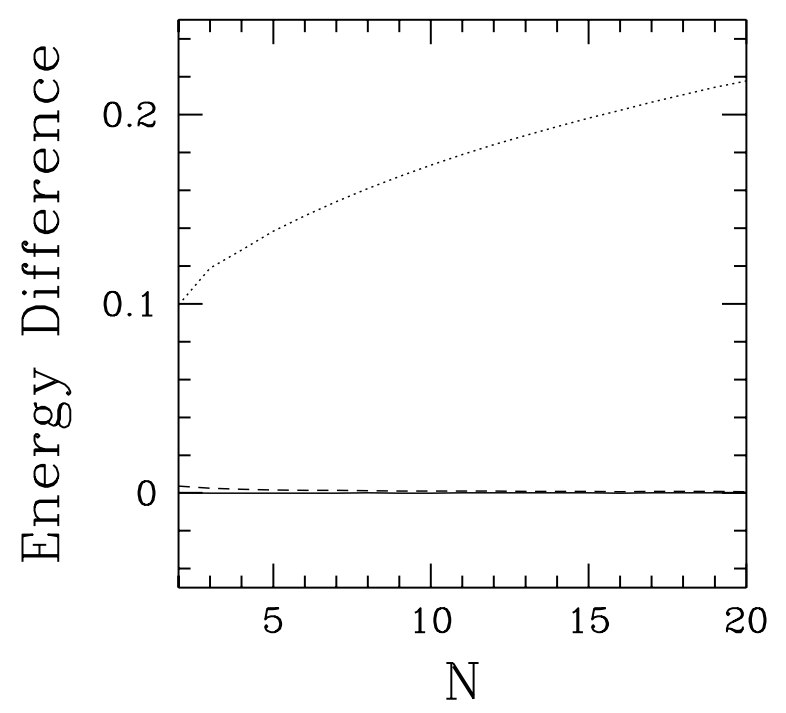

FIG. 2. Convergence of approximations to the quantum ground state energy for electron charge $e=0.5$. The curves are, from top to bottom, the error in the Strutinsky energy correction approach taken at zeroth, first, and second order: specifically $\Delta E \equiv E_{\mathrm{DFT}}-E_{\mathrm{GTF}}$ (dotted), $\Delta E-\Delta E^{(1)}$ (dashed), and $\Delta E-\Delta E^{(1)}-\Delta E^{(2)}$ (solid). The convergence in this case is excellent. 


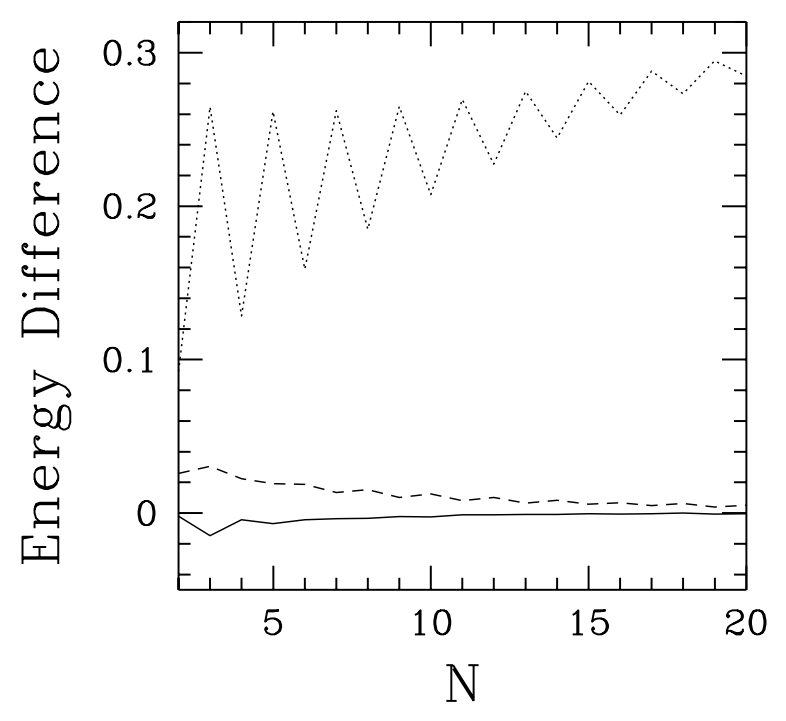

FIG. 3. Convergence of approximations to the quantum ground state energy for electron charge $e=1.0$. The curves are, from top to bottom, $\Delta E \equiv E_{\mathrm{DFT}}-E_{\mathrm{GTF}}$ (dotted), $\Delta E-\Delta E^{(1)}$ (dashed), and $\Delta E-\Delta E^{(1)}-\Delta E^{(2)}$ (solid).

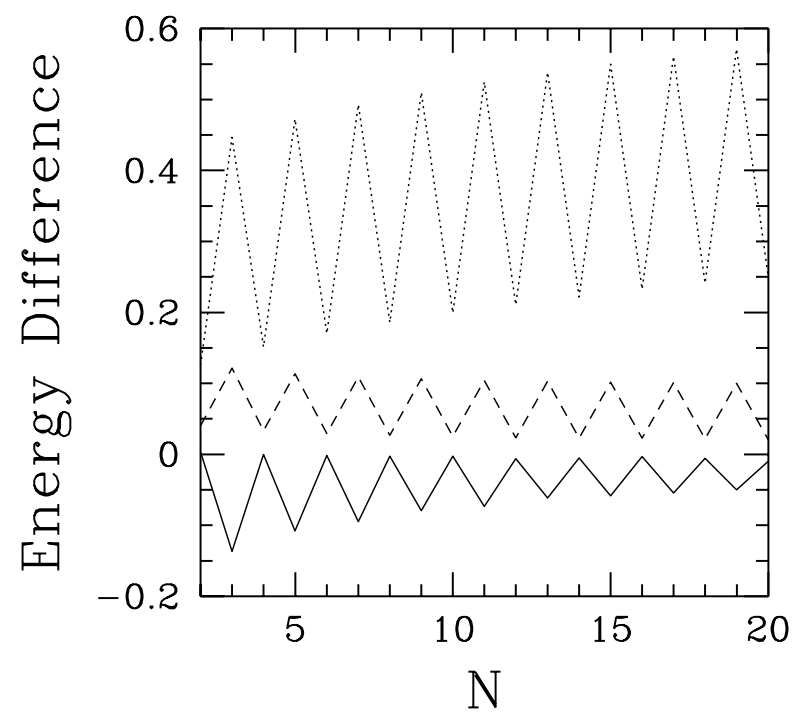

FIG. 4. Convergence of approximations to the quantum ground state energy for electron charge $e=1.5$. The curves are, from top to bottom, $\Delta E \equiv E_{\mathrm{DFT}}-E_{\mathrm{GTF}}$ (dotted), $\Delta E-\Delta E^{(1)}$ (dashed), and $\Delta E-\Delta E^{(1)}-\Delta E^{(2)}$ (solid). 


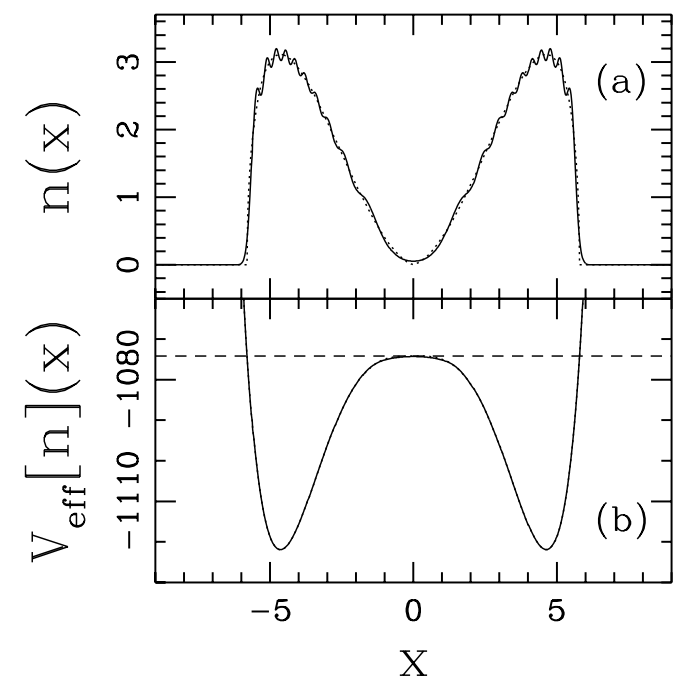

FIG. 5. The electron density (upper panel) and effective potential (lower panel) for $N=20$ particles and $e=1.5$. In the upper panel, results for both the Thomas-Fermi (dashed) and quantum (solid) cases are given. In the lower panel, the dashed horizontal line is the position of the chemical potential $\mu_{\mathrm{TF}}$.

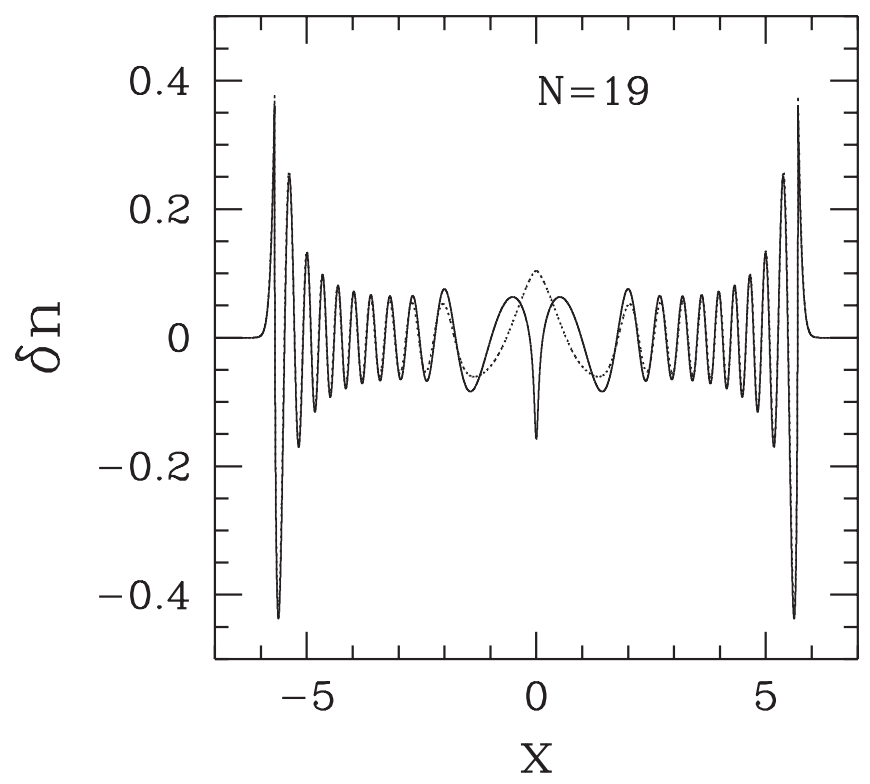

FIG. 6. Comparison between the exact $\delta n \equiv n_{\mathrm{DFT}}-n_{\mathrm{GTF}}$ (dashed line) and its approximation derived from Eq. (39) (solid line) for $e=1.5$ and $N=19$. 


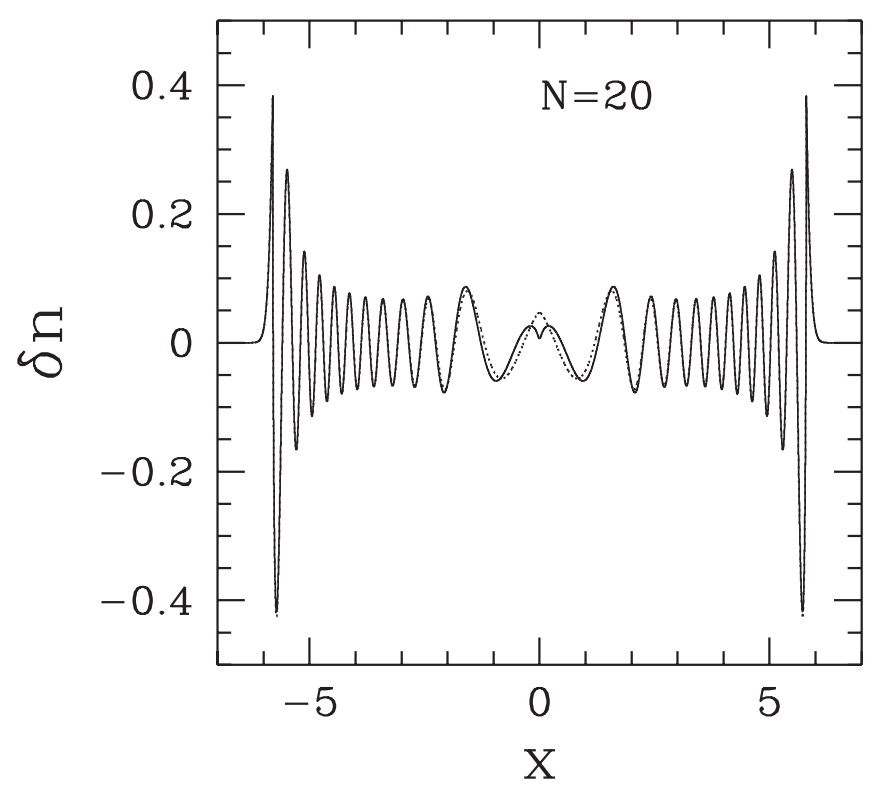

FIG. 7. Comparison between the exact $\delta n \equiv n_{\mathrm{DFT}}-n_{\mathrm{GTF}}$ (dashed line) and its approximation derived from Eq. (39) (solid line) for $e=1.5$ and $N=20$.

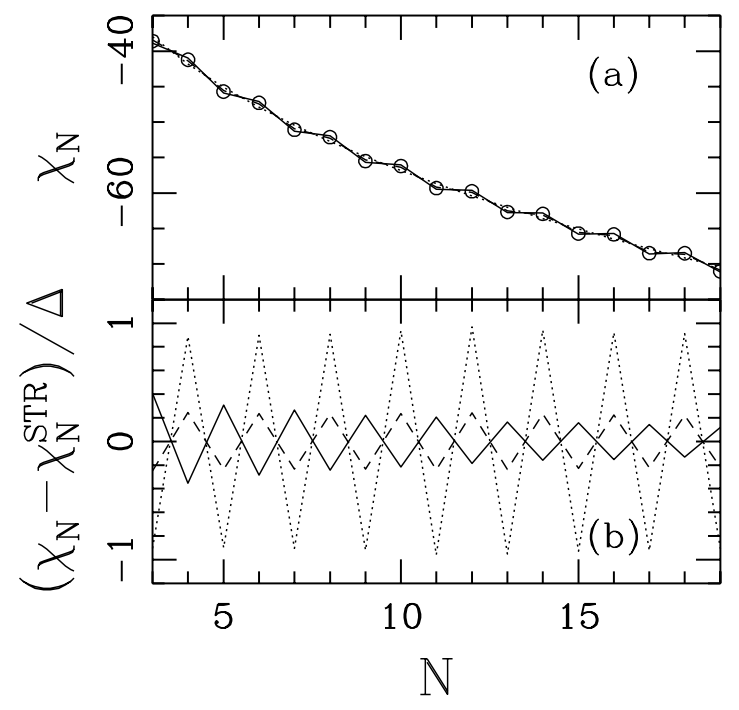

FIG. 8. The discrete inverse compressibility as a function of electron number $N$. The open circles are $\chi_{N}$ using the quantum ground state energy (exact). Results using three approximate ground state energies are shown: (1) dotted: Thomas-Fermi, $\chi_{N}^{\mathrm{STR}(0)}$ using $E_{\mathrm{GTF}}$, (2) dashed: first-order Strutinsky, $\chi_{N}^{\mathrm{STR}(1)}$ using $E_{\mathrm{GTF}}+\Delta E^{(1)}$, and (3) solid: second-order Strutinsky, $\chi_{N}^{\mathrm{STR}(2)}$ using $E_{\mathrm{GTF}}+\Delta E^{(1)}+\Delta E^{(2)}$. The lower panel shows the relative errors, $\left(\chi_{N}-\chi_{N}^{\mathrm{STR}}\right) / \Delta$, of the same three approximations. 